\title{
Analytic determinants and inverses of Toeplitz and Hankel tridiagonal matrices with perturbed columns
}

https://doi.org/10.1515/spma-2020-0012

Received February 13, 2020; accepted March 27, 2020

\begin{abstract}
In this paper, our main attention is paid to calculate the determinants and inverses of two types Toeplitz and Hankel tridiagonal matrices with perturbed columns. Specifically, the determinants of the $n \times n$ Toeplitz tridiagonal matrices with perturbed columns (type I, II) can be expressed by using the famous Fibonacci numbers, the inverses of Toeplitz tridiagonal matrices with perturbed columns can also be expressed by using the well-known Lucas numbers and four entries in matrix $\mathbb{A}$. And the determinants of the $n \times n$ Hankel tridiagonal matrices with perturbed columns (type I, II) are $(-1)^{\frac{n(n-1)}{2}}$ times of the determinant of the Toeplitz tridiagonal matrix with perturbed columns type I, the entries of the inverses of the Hankel tridiagonal matrices with perturbed columns (type I, II) are the same as that of the inverse of Toeplitz tridiagonal matrix with perturbed columns type I, except the position. In addition, we present some algorithms based on the main theoretical results. Comparison of our new algorithms and some recent works is given. The numerical result confirms our new theoretical results. And we show the superiority of our method by comparing the CPU time of some existing algorithms studied recently.
\end{abstract}

Keywords: Toeplitz tridiagonal matrices with perturbed columns, Hankel tridiagonal matrices with perturbed columns, Fibonacci number, Lucas number, determinant, inverse

\section{Introduction}

We start by introducing the main research object of this paper. An $n \times n$ Toeplitz tridiagonal matrix with perturbed columns $\mathbb{A}\left(c_{1}, a_{n} ; d,-d,-d ; a_{1}, c_{n}\right)$, shorted for $\mathbb{A}$, with $\mathbb{A}_{i, 1}=a_{i}$ for $i \in\{1, n\}, \mathbb{A}_{i, n}=c_{i}$ for $i \in\{1, n\}$ and $\mathbb{A}_{i, j}=-d$ for $j-1 \leq i \leq j(i \neq n), 2 \leq j \leq n, \mathbb{A}_{i, i-1}=d$ for $3 \leq i \leq n$ and 0 otherwise. This can be

\footnotetext{
Yaru Fu: School of Mathematics and Statistics, Linyi University, Linyi 276000, P. R. China, College of Information Technology, The University of Suwon, Wau-ri, Bongdam-eup, Hwaseong-si, Gyeonggi-do, 445-743, Korea, E-mail: tysffyr@163.com Xiaoyu Jiang: School of Information Science and Technology, Linyi University, Linyi 276000, P. R. China, E-mail: jxy19890422@sina.com

*Corresponding Author: Zhaolin Jiang: School of Mathematics and Statistics, Linyi University, Linyi 276000, P. R. China, Email: jzh1208@sina.com

Seongtae Jhang: College of Information Technology, The University of Suwon, Wau-ri, Bongdam-eup, Hwaseong-si, Gyeonggido, 445-743, Korea, E-mail: stjhang@suwon.ac.kr
} 
written as

$$
\mathbb{A}=\left(\begin{array}{ccccccc}
a_{1} & -d & 0 & \cdots & \cdots & 0 & c_{1} \\
0 & -d & \ddots & \ddots & & & 0 \\
0 & d & \ddots & \ddots & \ddots & & \vdots \\
\vdots & \ddots & \ddots & \ddots & \ddots & \ddots & \vdots \\
\vdots & & \ddots & \ddots & \ddots & \ddots & 0 \\
0 & & & \ddots & \ddots & -d & -d \\
a_{n} & 0 & \cdots & \cdots & 0 & d & c_{n}
\end{array}\right),
$$

where $d(d \neq 0), a_{1}, a_{n}, c_{1}$ and $c_{n}$ are arbitrary complex numbers.

In [1-4], the authors have studied Toeplitz matrices, general Hessenberg matrices and opposite-bordered tridiagonal matrix and so on. These matices have been usually applied in various application areas ranging from the computation of special functions to number theory [5], as well as in engineering, economics and heat conduction [6-8]. One could view an example that described the heat inside the material, such as the mixture energy conservation equation

$$
\int_{c S} \phi \rho_{g} h_{g} v_{g} d S+\int_{c S} \dot{q}^{\prime \prime} d S-\int_{c S} \rho h v_{c S} d S+\frac{d}{d t} \int_{c V} \rho e d V=0,
$$

where $\phi$ is the porosity of the solid, $\rho$ the density, $h$ the enthalpy, $v$ the velocity, $S$ the area, $e$ the energy, $V$ the volume and $\dot{q}^{\prime \prime}$ the heat flux according to Fourier's Law. Subscripts $c s, c v$ and $g$ are the control surface, the control volume and the gas phase, respectively. This equation can be discretized using a control volume finite element method [9] whose coefficient matrix $\mathbf{M}$ is an $n$-by- $n$ opposite-bordered tridiagonal matrix as follows

$$
\mathbf{M}=\left(\begin{array}{cccccc}
a_{1} & c_{1} & 0 & \cdots & 0 & h_{1} \\
d_{2} & a_{2} & c_{2} & \ddots & \vdots & h_{2} \\
g_{3} & \ddots & \ddots & \ddots & 0 & \vdots \\
\vdots & 0 & \ddots & \ddots & c_{n-2} & h_{n-2} \\
g_{n-1} & \vdots & \ddots & d_{n-1} & a_{n-1} & c_{n-1} \\
g_{n} & 0 & \ldots & 0 & d_{n} & a_{n}
\end{array}\right) .
$$

In addition, Eigenpairs, determinants and inverses of the tridiagonal matrices, anti-tridiagonal Hankel matrices, pentadiagonal matrices and cyclic pentadiagonal matrices with Toeplitz structure have been studied in [10-23].

The determinant and inversion of $\mathbb{A}\left(c_{1}, a_{n} ; d_{k}, a_{k}, c_{k} ; a_{1}, c_{n}\right)$ have been studied extensively and found with simple and analytic expression (see [24-26]). Recently, Jia proposed a new breakdown-free recursive algorithm for computing the determinants of periodic tridiagonal matrices via a three-term recurrence in [25]. In addition, an explicit formula for the determinant of the periodic tridiagonal matrix with Toeplitz structure is also discussed. In [26], the entries of the inverse of general periodic tridiagonal matrices are expressed in term of determinants whose values are related to the solutions of second order linear recurrences. However, when the entries of $\mathbb{A}\left(c_{1}, a_{n} ; d_{k}, a_{k}, c_{k} ; a_{1}, c_{n}\right)$ are rational numbers or algebraic quantities, the method is not available. Therefore, in [27], Hopkins and Kßlßç construct a new algorithm which need utilize a symbolic algebra (SA) system such as Maple or Mathematica. And the main idea of this method is to derive its LU factorization using backward continued fractions (BCF). Furthermore, many methods have been reported for solving systems of linear equations whose coefficient matrix is periodic tridiagonal, see [28-31].

This paper is motivated by the work of Jia and $\mathrm{Li}$ [4] who introduce the solution of opposite-bordered tridiagonal (OBT) systems of linear equations. They present two efficient algorithms which used reliable tridiagonal linear solver and column operation, respectively. And the computational accuracy and efficiency of the 
proposed algorithms are illustrated in the paper. Obviously, although the Toeplitz tridiagonal matrix with perturbed columns $\mathbb{A}$ is the special form of the opposite-bordered tridiagonal M. However, we can obtain much more efficient and simple theoretical results and algorithms for computing the determinants and inverses of Toeplitz tridiagonal matrices with perturbed columns. Therefore, it is meaningful to study the determinants and inverses of new matrices. Combining the Schur determinant formula and the special property of the upper triangular Toeplitz matrix, the determinant of the Toeplitz and Hankel tridiagonal matrices with perturbed columns can be obtained easily. Using the sparsity of the Toeplitz and Hankel tridiagonal matrices with perturbed columns, the famous Sherman-Morrison-Woodbury formula, the explicit expression of the inverse matrix can be obtained. And two efficient computational algorithms for main theoretical results are given and one example is also given to illustrate the algorithm.

Furthermore, we introduce two famous numbers, i.e. Fibonacci number and Lucas number, which satisfy the following recurrence relation [32]

$$
\begin{gathered}
F_{0}=0, F_{1}=1, F_{n}=F_{n-1}+F_{n-2}, F_{-n}=(-1)^{n+1} F_{n}(n \geq 2) . \\
L_{0}=2, L_{1}=1, L_{n}=L_{n-1}+L_{n-2}, L_{-n}=(-1)^{n} L_{n}(n \geq 2) .
\end{gathered}
$$

Notation. For convenience, let $\operatorname{det} \mathbb{A}$ denote the determinant of matrix $\mathbb{A}, \operatorname{circ}\left(x_{1}, x_{2}, \ldots, x_{n}\right)$ denote a circulant matrix whose rows are composed of cyclically shifted versions of the vector $\left(x_{1}, x_{2}, \ldots, x_{n}\right) . \hat{I}_{n}$ is the "reverse unit matrix", having ones along the secondary diagonal and zeros elsewhere.

The rest of this paper is organized as follows. We present the determinants of the Toeplitz and Hankel tridiagonal matrices with perturbed columns in the following section. In addition, the algorithm is presented for main theoretical result. Section 3 is devoted to computing the inverse matrices of the Toeplitz and Hankel tridiagonal matrices with perturbed columns, and the corresponding algorithm for the main theorem is given. In Section 4, one numerical example is given to show the performance of the new algorithm for the Toeplitz tridiagonal matrix with perturbed columns type I.

\section{The determinants of the Toeplitz and Hankel tridiagonal matrices with perturbed columns}

In this section, we obtain the explicit expression of the determinants of the Toeplitz and Hankel tridiagonal matrices with perturbed columns, respectively. The corresponding algorithm for computing the determinant of $\mathbb{A}$ is given. Moreover, complexity of this algorithm is compared with some existing algorithms.

Theorem 2.1. Let the $n \times n(n \geq 3)$ Toeplitz tridiagonal matrix with perturbed columns $\mathbb{A}$ be defined in (1). Then the determinant of $\mathbb{A}$ is

$$
\operatorname{det} \mathbb{A}=(-d)^{n-2}\left(a_{1} c_{n}-c_{1} a_{n}\right) F_{n-1}+(-d)^{n-1}\left[(-1)^{n-1} a_{n}+a_{1} F_{n-2}\right],
$$

where $F_{j}(j=n-2, n-1)$ are Fibonacci numbers.

Proof. Let $\mathcal{C}=\operatorname{circ}(0,1,0, \cdots, 0)$ be a permutation circulant matrix. Clearly,

$$
\operatorname{det} \mathcal{C}=(-1)^{n-3}
$$

and $\mathcal{C}$ is invertible. 
Then multiplying $\mathbb{A}$ by $\mathcal{C}$ from the right and dividing $\mathbb{A} \mathcal{C}$ into blocks as follows

$$
\begin{aligned}
\mathbb{A} C & =\left(\begin{array}{cc:cccccc}
c_{1} & a_{1} & -d & 0 & \cdots & \cdots & \cdots & 0 \\
0 & 0 & -d & -d & \ddots & & & \vdots \\
\hdashline & \ddots & d & -d & -d & \ddots & & \vdots \\
\vdots & & \ddots & \ddots & \ddots & \ddots & \ddots & \vdots \\
\vdots & & & \ddots & \ddots & \ddots & \ddots & 0 \\
0 & & & & \ddots & \ddots & \ddots & -d \\
-d & 0 & & & & \ddots & \ddots & -d \\
c_{n} & a_{n} & 0 & \cdots & \cdots & \cdots & 0 & d
\end{array}\right) \\
& =\left(\begin{array}{c:ccc}
\mathbb{A}_{11} & \mathbb{A}_{12} \\
\hdashline \mathbb{A}_{13} & \mathbb{A}_{14}
\end{array}\right),
\end{aligned}
$$

by taking determinants on both sides of the above equation and using the theorem in [33, p.10], we get

$$
\begin{aligned}
\operatorname{det} \mathbb{A} \operatorname{det} \mathcal{C} & =\operatorname{det}(\mathbb{A} \mathcal{C}) \\
& =\operatorname{det}\left(\mathbb{A}_{11}-\mathbb{A}_{12} \mathbb{A}_{14}^{-1} \mathbb{A}_{13}\right) \operatorname{det} \mathbb{A}_{14} .
\end{aligned}
$$

Now we devote our attention to computing the determinants of $\mathbb{A}_{14}, \mathbb{A}_{11}-\mathbb{A}_{12} \mathbb{A}_{14}^{-1} \mathbb{A}_{13}$. From the expression of $\mathbb{A}_{14}$ in $(7)$, we know that $\mathbb{A}_{14}$ is an $(n-2) \times(n-2)$ upper triangular Toeplitz matrix, therefore its determinant is

$$
\operatorname{det} \mathbb{A}_{14}=d^{n-2} \text {, }
$$

which implies that $\mathbb{A}_{14}$ is invertible and $\mathbb{A}_{14}^{-1}$ is also an upper triangular Toeplitz matrix (see [34], Lemma 2.5) with entries being $\frac{1}{d}$ time of Fibonacci numbers, i.e.

$$
\left(\mathbb{A}_{14}^{-1}\right)_{i, j}=\frac{F_{j-i+1}}{d}(-n+3 \leq i-j \leq 0)
$$

and otherwise is 0 , where $F_{j-i+1}$ is the $(j-i+1)$ th Fibonacci number. Hence, the explicit expression of the $\mathbb{A}_{11}-\mathbb{A}_{12} \mathbb{A}_{14}^{-1} \mathbb{A}_{13}$ is

$$
\mathbb{A}_{11}-\mathbb{A}_{12} \mathbb{A}_{14}^{-1} \mathbb{A}_{13}=\left(\begin{array}{cc}
c_{1}-d F_{n-3}+c_{n} F_{n-2} & a_{1}+a_{n} F_{n-2} \\
c_{n} F_{n-1}-d F_{n-2} & a_{n} F_{n-1}
\end{array}\right),
$$

then based on the recursive relationship of Fibonacci numbers, the determinant of $\mathbb{A}_{11}-\mathbb{A}_{12} \mathbb{A}_{14}^{-1} \mathbb{A}_{13}$ can be expressed as

$$
\operatorname{det}\left(\mathbb{A}_{11}-\mathbb{A}_{12} \mathbb{A}_{14}^{-1} \mathbb{A}_{13}\right)=d\left[(-1)^{n-1} a_{n}+a_{1} F_{n-2}\right]+F_{n-1}\left(c_{1} a_{n}-a_{1} c_{n}\right)
$$

Finally, substituting (6), (9) and (10) into (8), we get (5), which completes the proof.

The resulting algorithm is summarized as follows.

Algorithm 1. Compute the determinant of the Toeplitz tridiagonal matrix with perturbed columns $\mathbb{A}$ defined in (1).

Step 1. Input the components $a_{1}, a_{n}, c_{1}, c_{n}, d$, order $n$ and generate Fibonacci numbers $F_{i}(i=n-2, n-1)$ by (3).

Step 2. Calculate the determinant of the perturbed Toeplitz tridiagonal matrix with perturbed columns $\mathbb{A}$ by (5).

Step 3. Output the determinant of matrix $\mathbb{A}$ : $\operatorname{det} \mathbb{A}$. 
From the above algorithm, we know that Algorithm 1 requires $4 n+14$ operations for computing the determinant of the matrix $\mathbb{A}$. The complexity of our method can be reduced to $O(\log n)$ according to the analysis in [36, p.226-227]. Then we compare the computational cost between LU decomposition and Algorithm 1 in Table 1. In Table 1, the LU decomposition for computing the determinant of $\mathbb{A}$ refers to simplifying the general LU decomposition according to the special matrix proposed in this paper. Comparing the results in Table 1, we can see that the computational cost of our algorithm is less than that of the LU decomposition algorithm.

Table 1: The complexity comparison of computing determinant for different algorithms.

\begin{tabular}{c|c}
\hline Algorithm & Complexity \\
\hline \hline LU decomposition & $14 n-15$ \\
\hline Algorithm 1 & $4 n+14$ \\
\hline
\end{tabular}

Theorem 2.2. An $n \times n(n \geq 3)$ Toeplitz tridiagonal matrix with perturbed columns $\mathbb{B}$ (type $\mathrm{II})$ is such a matrix with $\mathbb{B}=\hat{I}_{n} \mathbb{A} \hat{I}_{n}$, then the determinant of $\mathbb{B}$ is

$$
\operatorname{det} \mathbb{B}=(-d)^{n-2}\left(a_{1} c_{n}-c_{1} a_{n}\right) F_{n-1}+(-d)^{n-1}\left[(-1)^{n-1} a_{n}+a_{1} F_{n-2}\right],
$$

where $\mathbb{A}$ is the same as (1), $F_{j}(j=n-2, n-1)$ are Fibonacci numbers.

Proof. We can get (11) by using $\operatorname{det} \mathbb{B}=\operatorname{det} \hat{I}_{n} \operatorname{det} \mathbb{A} \operatorname{det} \hat{I}_{n}=\operatorname{det} \mathbb{A}$ and Theorem 2.1.

Theorem 2.3. Let $\widehat{\mathbb{C}}:=\hat{I}_{n} \mathbb{A}$ be an $n \times n(n \geq 3)$ Hankel tridiagonal matrix with perturbed columns type $\mathrm{I}$. Then we have

$$
\begin{aligned}
\operatorname{det} \hat{\mathbb{C}} & =(-1)^{\frac{n(n-1)}{2}} \times \\
& \left\{(-d)^{n-2}\left(a_{1} c_{n}-c_{1} a_{n}\right) F_{n-1}+(-d)^{n-1}\left[(-1)^{n-1} a_{n}+a_{1} F_{n-2}\right]\right\},
\end{aligned}
$$

where $\mathbb{A}$ is defined in (1), $F_{j}(j=n-2, n-1)$ are Fibonacci numbers.

Proof. Since

$$
\operatorname{det} \hat{\mathbb{C}}=\operatorname{det} \hat{I}_{n} \operatorname{det} \mathbb{A},
$$

by using Theorem 2.1, the conclusion holds.

Theorem 2.4. An $n \times n(n \geq 3)$ Hankel tridiagonal matrix with perturbed columns $\mathbb{D}$ (type $\mathrm{II})$ is such a matrix with $\mathbb{D}=\mathbb{A} \hat{I}_{n}$, then we get

$$
\begin{aligned}
\operatorname{det} \mathbb{D} & =(-1)^{\frac{n(n-1)}{2}} \times \\
& \left\{(-d)^{n-2}\left(a_{1} c_{n}-c_{1} a_{n}\right) F_{n-1}+(-d)^{n-1}\left[(-1)^{n-1} a_{n}+a_{1} F_{n-2}\right]\right\},
\end{aligned}
$$

where $\mathbb{A}$ is given in (1), $F_{j}(j=n-2, n-1)$ are Fibonacci numbers.

Proof. Through the Theorem 2.1 and equation $\operatorname{det} \mathbb{D}=\operatorname{det} \hat{I}_{n} \operatorname{det} \mathbb{A}$, we can obtain (13).

The corresponding algorithms for computing the determinants of $\mathbb{B}, \hat{\mathbb{C}}$ and $\mathbb{D}$ can be easily given according to the Theorem 2.2, Theorem 2.3, Theorem 2.4 and Algorithm 1, respectively. 


\section{The inverses of the Toeplitz and Hankel tridiagonal matrices with perturbed columns}

In this section, our attention is devoted to computing the inverse matrices of $\mathbb{A}, \mathbb{B}, \hat{\mathbb{C}}$ and $\mathbb{D}$. The corresponding algorithm for computing the inverse matrix of $\mathbb{A}$ is presented.

Theorem 3.1. Suppose that an $n \times n(n \geq 3)$ matrix $\mathbb{A}$ has the same form as (1) and $\mathbb{A}$ is invertible. Then its inverse matrix can be expressed as

$$
\mathbb{A}^{-1}=\left(\alpha_{i, j}\right)_{n \times n},
$$

where

$$
\alpha_{i, j}= \begin{cases}\frac{\left[(-1)^{n} L_{n}^{2}+1\right] c_{n}+\left(\hat{e}_{1}+3\right) d}{\left[(-1)^{n} L_{n}^{2}+1\right] \hat{e}_{2}+\left(\hat{e}_{1}+3\right) a_{1} d+d a_{n}\left(L_{n}+L_{n+2}\right)}, & i=j=1, \\ \frac{(-1)^{n+1} c_{1}\left(L_{n}+L_{n+2}\right)+\left(\hat{e}_{1}+3\right) c_{n}+\left[(-1)^{n} L_{n-1}^{2}+9\right] d}{\left[(-1)^{n} L_{n}^{2}+1\right] \hat{e}_{2}+\left(\hat{e}_{1}+3\right) a_{1} d+d a_{n}\left(L_{n}+L_{n+2}\right)}, & i=1, j=2, \\ \frac{-a_{n}\left(L_{n}+L_{n+2}\right)}{\left[(-1)^{n} L_{n}^{2}+1\right] \hat{e}_{2}+\left(\hat{e}_{1}+3\right) a_{1} d+d a_{n}\left(L_{n}+L_{n+2}\right)}, & i=2, j=1, \\ \frac{\left(\hat{e}_{1}+3\right) \hat{e}_{2}+\left[9+(-1)^{n} L_{n-1}^{2}\right] d a_{1}}{\left(\hat{e}_{1}+3\right) d^{2} a_{1}+d^{2} a_{n}\left(L_{n}+L_{n+2}\right)+\left((-1)^{n} L_{n}^{2}+1\right] \hat{e}_{2} d}, & i=j=2, \\ \frac{1}{d}+\alpha_{2,1}+\alpha_{2,2}, & i=2, j=3, \\ -\alpha_{2,2}-\frac{1}{d}, & i=3, j=2, \\ \alpha_{i, j-1}+\alpha_{i, j-2}, & i=1,3 \leq j \leq n, \\ \alpha_{i, j-1}+\alpha_{i, j-2}, & i=2,4 \leq j \leq n, \\ -\alpha_{2, j}, & i=3,1 \leq j \leq n, j \neq 2, \\ \alpha_{i-2, j}-\alpha_{i-1, j}, & 4 \leq i \leq n, j=1,2, \\ \alpha_{i, 1}+\alpha_{i, 2}, & 4 \leq i \leq n, j=3, \\ \alpha_{i, j-1}+\alpha_{i, j-2}, & 4 \leq j \leq i \leq n, \\ \alpha_{i-2, j}-\alpha_{i-1, j}, & 4 \leq i<j \leq n\end{cases}
$$

with

$$
\begin{aligned}
& \hat{e}_{1}=(-1)^{n+1} L_{n-1} L_{n}, \\
& \hat{e}_{2}=a_{1} c_{n}-c_{1} a_{n},
\end{aligned}
$$

$L_{j}(j=n-1, n, n+2)$ are Lucas numbers.

Proof. For every matrix, it is true that

$$
\mathbb{A}^{-1} \mathbb{A}=\mathbb{A A}^{-1}=I,
$$

where $I$ denotes the $n \times n$ identity matrix.

1. For $\mathbb{A}^{-1}=I$,

a. when $i=2,1 \leq j \leq n$, we know that

$$
-\alpha_{2, j} d-\alpha_{3, j} d=\delta_{i, j},
$$

where $\delta_{i, j}$ is the Kronecter symbol which is equal to 1 if $i=j$ and 0 if $i \neq j$.

If $i=j=2$, note that $\delta_{i, j}=1$ and $d \neq 0$, then

$$
\alpha_{3,2}=-\alpha_{2,2}-\frac{1}{d} .
$$

If $j \neq 2$, for every $1 \leq j \leq n$, note that $\delta_{i, j}=0$ and $d \neq 0$, then

$$
\alpha_{3, j}=-\alpha_{2, j}
$$


b. when $3 \leq i \leq n-1,1 \leq j \leq n$, we obtain

$$
\alpha_{i-1, j} d-\alpha_{i, j} d-\alpha_{i+1, j} d=\delta_{i, j} .
$$

If $j=1,2$, for every $3 \leq i \leq n-1$, note that $\delta_{i, j}=0$ and $d \neq 0$, then

$$
\alpha_{i, j}=\alpha_{i-2, j}-\alpha_{i-1, j}(4 \leq i \leq n)
$$

If $3 \leq j \leq n, 3 \leq i \leq n-1$ and $i<j$, note that $\delta_{i, j}=0$ and $d \neq 0$, then

$$
\alpha_{i, j}=\alpha_{i-2, j}-\alpha_{i-1, j}(4 \leq i<j \leq n) .
$$

2. For $\mathbb{A}^{-1} \mathbb{A}=I$, when $1 \leq i \leq n, 2 \leq j \leq n-1$, we get

$$
-\alpha_{i, j-1} d-\alpha_{i, j} d+\alpha_{i, j+1} d=\delta_{i, j}
$$

If $i=1$, for every $2 \leq j \leq n-1$, note that $\delta_{1, j}=0$ and $d \neq 0$, then

$$
\alpha_{1, j}=\alpha_{1, j-1}+\alpha_{1, j-2}(3 \leq j \leq n) .
$$

If $i=j=2$, note that $\delta_{i, j}=1$ and $d \neq 0$, then

$$
\alpha_{2,3}=\frac{1}{d}+\alpha_{2,1}+\alpha_{2,2} .
$$

If $i=2$, for every $3 \leq j \leq n-1$, note that $\delta_{2, j}=0$ and $d \neq 0$, then

$$
\alpha_{2, j}=\alpha_{2, j-1}+\alpha_{2, j-2}(4 \leq j \leq n) .
$$

If $4 \leq i \leq n, j=2$, note that $\delta_{i, j}=0$ and $d \neq 0$, then

$$
\alpha_{i, 3}=\alpha_{i, 2}+\alpha_{i, 1}(4 \leq i \leq n) .
$$

If $3 \leq i \leq n, 3 \leq j \leq n-1$ and $j<i$, note that $\delta_{i, j}=0$ and $d \neq 0$, then

$$
\alpha_{i, j}=\alpha_{i, j-1}+\alpha_{i, j-2}(4 \leq j \leq i \leq n) .
$$

In order to obtain the inverse of matrix $\mathbb{A}$, from the above discussion, we only need to calculate the four entries

$$
\alpha_{1,1}, \alpha_{1,2}, \alpha_{2,1}, \alpha_{2,2}
$$

to explicitly express its inverse matrix $\mathbb{A}^{-1}$.

In the following, we devote our attention to computing these four entries. Firstly, we divide the matrix $\mathbb{A}$ into the following form

$$
\mathbb{A}=d \mathbb{L}+\mathbb{Q E},
$$

where

$$
\mathbb{L}=\left(\begin{array}{ccccccc}
\frac{L_{n-1}+L_{n+1}}{L_{n}+L_{n+2}} & -1 & 0 & \cdots & \cdots & 0 & \frac{5(-1)^{n+1}}{L_{n}+L_{n+2}} \\
1 & -1 & \ddots & \ddots & & & 0 \\
0 & \ddots & \ddots & \ddots & \ddots & & \vdots \\
\vdots & \ddots & \ddots & \ddots & \ddots & \ddots & \vdots \\
\vdots & & \ddots & \ddots & \ddots & \ddots & 0 \\
0 & & & \ddots & \ddots & -1 & -1 \\
\frac{L_{n}+L_{n+2}}{L_{n}} & 0 & \cdots & \cdots & 0 & 1 & \frac{L_{n-1}+L_{n+1}}{L_{n}+L_{n+2}}
\end{array}\right),
$$




$$
\mathbb{Q}=\left(\begin{array}{cc}
a_{1}-\frac{d\left(L_{n-1}+L_{n+1}\right)}{L_{n}+L_{n+2}} & c_{1}-\frac{5 d(-1)^{n+1}}{L_{n}+L_{n+2}} \\
-d & 0 \\
0 & \vdots \\
\vdots & \vdots \\
0 & 0 \\
a_{n}-\frac{5 d}{L_{n}+L_{n+2}} & c_{n}-\frac{d\left(L_{n-1}+L_{n+1}\right)}{L_{n}+L_{n+2}}
\end{array}\right)_{n \times 2},
$$

and

$$
\mathbb{E}=\left(\begin{array}{lllll}
1 & 0 & \cdots & 0 & 0 \\
0 & 0 & \cdots & 0 & 1
\end{array}\right)_{2 \times n}
$$

According to the Sherman-Morrison-Woodbury formula (see [35, p.50]), we yields

$$
\begin{aligned}
A^{-1} & =(d \mathbb{L}+\mathbb{Q E})^{-1} \\
& =\frac{1}{d} \mathbb{L}^{-1}-\frac{1}{d^{2}} \mathbb{L}^{-1} \mathbb{Q}\left(I+\frac{1}{d} \mathbb{E} \mathbb{L}^{-1} \mathbb{Q}\right)^{-1} \mathbb{E} \mathbb{L}^{-1},
\end{aligned}
$$

Now we turn to compute these matrices $\mathbb{L}^{-1} \mathbb{Q}, \mathbb{E L}^{-1},\left(I+\frac{1}{d} \mathbb{E}^{-1} \mathbb{Q}\right)^{-1}$. From (18), it is easy to verify that

$$
\mathbb{L}^{-1}=\frac{1}{5}\left(l_{i, j}\right)_{n \times n},
$$

where

$$
l_{i, j}= \begin{cases}L_{j-i+1}, & -n+1 \leq i-j \leq 0, \\ -L_{-(i-j+1)}, & 0<i-j \leq n-1 .\end{cases}
$$

Multiplying $\mathbb{L}^{-1}$ by $\mathbb{E}$ from the left, we obtain

$$
\mathbb{E}^{-1}=\frac{1}{5}\left(\begin{array}{ccccccc}
L_{1} & L_{2} & \cdots & \cdots & L_{n-2} & L_{n-1} & L_{n} \\
-L_{-n} & -L_{-(n-1)} & \cdots & \cdots & -L_{-3} & -L_{-2} & L_{1}
\end{array}\right)_{2 \times n},
$$

and multiplying $\mathbb{L}^{-1}$ by $\mathbb{Q}$ from the right, denoting $\hat{\mathbb{F}}=\mathbb{L}^{-1} \mathbb{Q}$, we have

$$
\hat{\mathbb{F}}=\frac{1}{5}\left(\hat{f}_{i, j}\right)_{n \times 2}= \begin{cases}a_{1}+L_{n} a_{n}-5 d, & i=1, j=1, \\ l_{i, 1} a_{1}+l_{i, n} a_{n}, & 2 \leq i \leq n, j=1, \\ l_{i, 1} c_{1}+l_{i, n} c_{n}-L_{n-i} d, & 1 \leq i \leq n, j=2 .\end{cases}
$$

Then computing the $2 \times 2$ matrix $I+\frac{1}{d} \mathbb{E} \mathbb{L}^{-1} \mathbb{Q}$ by (20) and (25), i.e.

$$
I+\frac{1}{d} \mathbb{E} \mathbb{L}^{-1} \mathbb{Q}=\frac{1}{5 d}\left(\begin{array}{cc}
\hat{f}_{1,1}+5 d & \hat{f}_{1,2} \\
\hat{f}_{n, 1} & \hat{f}_{n, 2}+5 d
\end{array}\right),
$$

and its inverse matrix is

$$
\left(I+\frac{1}{d} \mathbb{E} \mathbb{L}^{-1} \mathbb{Q}\right)^{-1}=\frac{5 d}{\hat{r}}\left(\begin{array}{cc}
\hat{f}_{n, 2}+5 d & -\hat{f}_{1,2} \\
-\hat{f}_{n, 1} & \hat{f}_{1,1}+5 d
\end{array}\right),
$$

where $\hat{r}$ is the determinant of matrix

$$
\left(\begin{array}{cc}
\hat{f}_{1,1}+5 d & \hat{f}_{1,2} \\
\hat{f}_{n, 1} & \hat{f}_{n, 2}+5 d
\end{array}\right)
$$

and

$$
\begin{aligned}
& \hat{r}=\left[(-1)^{n} L_{n}^{2}+1\right] \hat{e}_{2}+\left(\hat{e}_{1}+3\right) a_{1} d+d a_{n}\left(L_{n}+L_{n+2}\right), \\
& \hat{e}_{1}=(-1)^{n+1} L_{n-1} L_{n}, \quad \hat{e}_{2}=a_{1} c_{n}-c_{1} a_{n} .
\end{aligned}
$$


Following, multiplying $\left(I+\frac{1}{d} \mathbb{E} \mathbb{L}^{-1} \mathbb{Q}\right)^{-1}$ by $\mathbb{L}^{-1} \mathbb{Q}$ and $\mathbb{E} L^{-1}$ from the left and right, respectively, denoting $\hat{\mathbb{G}}=\mathbb{L}^{-1} \mathbb{Q}\left(I+\frac{1}{d} \mathbb{E} \mathbb{L}^{-1} \mathbb{Q}\right)^{-1} \mathbb{E} \mathbb{L}^{-1}$, we have

$$
\hat{\mathbb{G}}=\frac{d}{5 \hat{r}}\left(\hat{g}_{i, j}\right)_{n \times n},
$$

where

$$
\hat{g}_{i, j}=l_{1, j}\left[\hat{f}_{i, 1}\left(\hat{f}_{n, 2}+5 d\right)-\hat{f}_{i, 2} \hat{f}_{n, 1}\right]+l_{n, j}\left[\hat{f}_{i, 2}\left(\hat{f}_{1,1}+5 d\right)-\hat{f}_{i, 1} \hat{f}_{1,2}\right],
$$

and $l_{1, j}, l_{n, j} \hat{f}_{i, 1}$ and $\hat{f}_{i, 2}$ are defined in (23) and (25), respectively. Substituting (22) and (30) into (21), we obtain

$$
\left(\mathbb{A}^{-1}\right)_{i, j}=\frac{l_{i, j}}{5 d}-\frac{\hat{g}_{i, j}}{5 d \hat{r}} \triangleq \alpha_{i, j},
$$

thus based on the recursive relationship of Lucas numbers and substituting (23), (25), (28), (30) into (31), we have

$$
\begin{aligned}
& \alpha_{1,1}=\frac{l_{1,1} \hat{r}-\hat{g}_{1,1}}{5 d \hat{r}}=\frac{\left[(-1)^{n} L_{n}^{2}+1\right] c_{n}+\left(\hat{e}_{1}+3\right) d}{\left[(-1)^{n} L_{n}^{2}+1\right] \hat{e}_{2}+\left(\hat{e}_{1}+3\right) a_{1} d+d a_{n}\left(L_{n}+L_{n+2}\right)}, \\
& \alpha_{1,2}=\frac{l_{1,2} \hat{r}-\hat{g}_{1,2}}{5 d \hat{r}}=\frac{(-1)^{n+1} c_{1}\left(L_{n}+L_{n+2}\right)+\left(\hat{e}_{1}+3\right) c_{n}+\left[(-1)^{n} L_{n-1}^{2}+9\right] d}{\left[(-1)^{n} L_{n}^{2}+1\right] \hat{e}_{2}+\left(\hat{e}_{1}+3\right) a_{1} d+d a_{n}\left(L_{n}+L_{n+2}\right)}, \\
& \alpha_{2,1}=\frac{l_{2,1} \hat{r}-\hat{g}_{2,1}}{5 d \hat{r}}=\frac{-a_{n}\left(L_{n}+L_{n+2}\right)}{\left[(-1)^{n} L_{n}^{2}+1\right] \hat{e}_{2}+\left(\hat{e}_{1}+3\right) a_{1} d+d a_{n}\left(L_{n}+L_{n+2}\right)}, \\
& \alpha_{2,2}=\frac{l_{2,2} \hat{r}-\hat{g}_{2,2}}{5 d \hat{r}}=\frac{\left(\hat{e}_{1}+3\right) \hat{e}_{2}+\left[9+(-1)^{n} L_{n-1}^{2}\right] d a_{1}}{\left(\hat{e}_{1}+3\right) d^{2} a_{1}+d^{2} a_{n}\left(L_{n}+L_{n+2}\right)+\left[(-1)^{n} L_{n}^{2}+1\right] \hat{e}_{2} d},
\end{aligned}
$$

where $\hat{e}_{1}$ and $\hat{e}_{2}$ are the same as (28). Hence the conclusion holds.

The algorithm for computing the inverse matrix of $\mathbb{A}$ is described as follows.

Algorithm 2 Compute the inverse of the Toeplitz tridiagonal matrix with perturbed columns $\mathbb{A}$ defined in (1). Step 1. Input $a_{1}, a_{n}, c_{1}, c_{n}, d$, order $n$ and generate Lucas numbers $L_{i}(i=n-1, n, n+2)$ by (4).

Step 2. Calculate the four entries $\alpha_{1,1}, \alpha_{1,2}, \alpha_{2,1}, \alpha_{2,2}$ via (14) and (15).

Step 3. Calculate the remaining entries of $\alpha_{i, j}$ by (14).

Step 4. Output the inverse matrix $\mathbb{A}^{-1}$.

The computational cost (i.e., the number of basic arithmetic operations) for Algorithm 2 is $n^{2}+2 n+44$. The comparison of complexity which is listed in Table 2, where the complexity of the LU decomposition is also presented. Here, the LU decomposition refers to simplifying the general LU decomposition for computing the inverse of general matrix according to the special matrix proposed in this paper. From Table 2, we can see that the computational cost of our algorithm is much less than that of the LU decomposition algorithm.

Table 2: The complexity comparison of computing inverse for different algorithms.

\begin{tabular}{c|c}
\hline Algorithm & Complexity \\
\hline \hline LU decomposition & $2 n^{2}+23 n-34$ \\
\hline Algorithm 2 & $n^{2}+2 n+44$ \\
\hline
\end{tabular}

Theorem 3.2. Assume that the Toeplitz tridiagonal matrix with perturbed columns $\mathbb{B}$ is invertible, then its inverse matrix can be expressed as

$$
\mathbb{B}^{-1}=\left(\hat{b}_{i, j}\right)_{n \times n}=\left(\alpha_{n+1-i, n+1-j}\right)_{n \times n}(n \geq 3),
$$

where $\alpha_{i, j}(1 \leq i, j \leq n)$ are given in (14). 
Proof. We can get (32) by using $\mathbb{B}^{-1}=\hat{I}_{n}^{-1} \mathbb{A}^{-1} \hat{I}_{n}^{-1}=\hat{I}_{n} \mathbb{A}^{-1} \hat{I}_{n}$ and Theorem 3.1.

Theorem 3.3. Assume that the $n \times n(n \geq 3)$ Hankel tridiagonal matrix with perturbed columns $\hat{\mathbb{C}}$ is invertible, then we have

$$
\hat{\mathbb{C}}^{-1}=\left(\hat{c}_{i, j}\right)_{n \times n}=\left(\alpha_{i, n+1-j}\right)_{n \times n},
$$

where $\alpha_{i, j}(1 \leq i, j \leq n)$ are the same as (14).

Proof. Since

$$
\hat{\mathbb{C}}^{-1}=\mathbb{A}^{-1} \hat{I}_{n}^{-1}=\mathbb{A}^{-1} \hat{I}_{n},
$$

by using Theorem 3.1, the conclusion holds.

Theorem 3.4. Assume that the $n \times n(n \geq 3)$ Hankel tridiagonal matrix with perturbed columns $\mathbb{D}$ is invertible, then we obtain

$$
\mathbb{D}^{-1}=\left(\hat{d}_{i, j}\right)_{n \times n}=\left(\alpha_{n+1-i, j}\right)_{n \times n},
$$

where $\alpha_{i, j}(1 \leq i, j \leq n)$ are defined in (14).

Proof. Through the Theorem 3.1 and equation $\mathbb{D}^{-1}=\hat{I}_{n}^{-1} \mathbb{A}^{-1}=\hat{I}_{n} \mathbb{A}^{-1}$, we can obtain (34).

One could propose the corresponding algorithms for inverse matrices of $\mathbb{B}, \hat{\mathbb{C}}$ and $\mathbb{D}$ by using Theorem 3.2, Theorem 3.3, Theorem 3.4 and Algorithm 2, respectively.

\section{Example}

In this section, an example is given to demonstrate the correctness and performance of the method. In the numerical experiment, $n$ is the order of matrix, "-" denotes exceeding the memory of MATLAB. We report the running time in seconds (denoted by CPU time). And the CPU time computed by the average time of 100 consecutive executions. The MATLAB function 'det' is used to compute the determinant in MATLAB. And the experiment is performed on a double-precision PC with a MATLAB (R2018a) and a central processor of 3.40 GHz [Intel(R) Core(TM)i7-6700 CPU], 8GB Microsoft Windows 10 operating system.

Example 4.1 In this example, we consider the following $n$-by- $n$ Toeplitz tridiagonal matrix with perturbed columns

$$
\left(\begin{array}{ccccccc}
2 & -1 & 0 & \cdots & \cdots & \cdots & 1 \\
0 & -1 & -1 & \ddots & & & \vdots \\
0 & 1 & \ddots & \ddots & \ddots & & \vdots \\
\vdots & \ddots & \ddots & \ddots & \ddots & \ddots & \vdots \\
\vdots & & \ddots & \ddots & \ddots & -1 & 0 \\
\vdots & & & \ddots & 1 & -1 & -1 \\
1 & \cdots & \cdots & \cdots & 0 & 1 & 1
\end{array}\right)_{n \times n} .
$$

For this example, the correctness and CPU time of the Algorithm 1, the method in MATLAB and LU decomposition are presented in Table 3. And a brief comparison for CPU time of each algorithm is showed in Figure 1. When the order of the matrix is much more than 1476, we can not get the precise value of the determinant because of the memory of MATLAB. From Table 3, we can see that the determinant of each method is same. However, the CPU time of our method is much little than other methods for each order. This result is reasonable because the complexity of new algorithm is very low. 
Table 3: The CPU time comparison for computing the determinant of Algorithm 1, the method in MATLAB and LU decomposition.

\begin{tabular}{|c|c|c|c|}
\hline$n$ & Algorithm & Determinant & CPU time (s) \\
\hline 1000 & $\begin{array}{c}\text { MATLAB(det) } \\
\text { LU decomposition } \\
\text { Algorithm } 1\end{array}$ & $\begin{array}{l}-6.3417 e+207 \\
-6.3417 e+207 \\
-6.3417 e+207\end{array}$ & $\begin{array}{l}1.8100 \mathrm{e}-02 \\
1.1000 \mathrm{e}-02 \\
6.1686 \mathrm{e}-04\end{array}$ \\
\hline 1100 & $\begin{array}{c}\text { MATLAB(det) } \\
\text { LU decomposition } \\
\text { Algorithm } 1\end{array}$ & $\begin{array}{l}-5.0231 e+228 \\
-5.0231 e+228 \\
-5.0231 e+228\end{array}$ & $\begin{array}{l}1.8500 \mathrm{e}-02 \\
1.2500 \mathrm{e}-02 \\
5.1530 \mathrm{e}-04\end{array}$ \\
\hline 1200 & $\begin{array}{c}\text { MATLAB(det) } \\
\text { LU decomposition } \\
\text { Algorithm } 1\end{array}$ & $\begin{array}{l}-3.9786 e+249 \\
-3.9786 e+249 \\
-3.9786 e+249\end{array}$ & $\begin{array}{l}2.2800 \mathrm{e}-02 \\
1.1200 \mathrm{e}-02 \\
6.5142 \mathrm{e}-04\end{array}$ \\
\hline 1300 & $\begin{array}{c}\text { MATLAB(det) } \\
\text { LU decomposition } \\
\text { Algorithm } 1\end{array}$ & $\begin{array}{l}-3.1514 \mathrm{e}+270 \\
-3.1514 \mathrm{e}+270 \\
-3.1514 \mathrm{e}+270\end{array}$ & $\begin{array}{l}2.8900 \mathrm{e}-02 \\
1.2600 \mathrm{e}-02 \\
4.6603 \mathrm{e}-04\end{array}$ \\
\hline 1400 & $\begin{array}{c}\text { MATLAB(det) } \\
\text { LU decomposition } \\
\text { Algorithm } 1\end{array}$ & $\begin{array}{l}-2.4961 e+291 \\
-2.4961 e+291 \\
-2.4961 e+291\end{array}$ & $\begin{array}{l}3.5400 \mathrm{e}-02 \\
1.0500 \mathrm{e}-02 \\
4.9307 \mathrm{e}-04\end{array}$ \\
\hline 1500 & $\begin{array}{c}\text { MATLAB(det) } \\
\text { LU decomposition } \\
\text { Algorithm } 1\end{array}$ & $\begin{array}{l}- \\
- \\
-\end{array}$ & $\begin{array}{l}- \\
- \\
-\end{array}$ \\
\hline
\end{tabular}

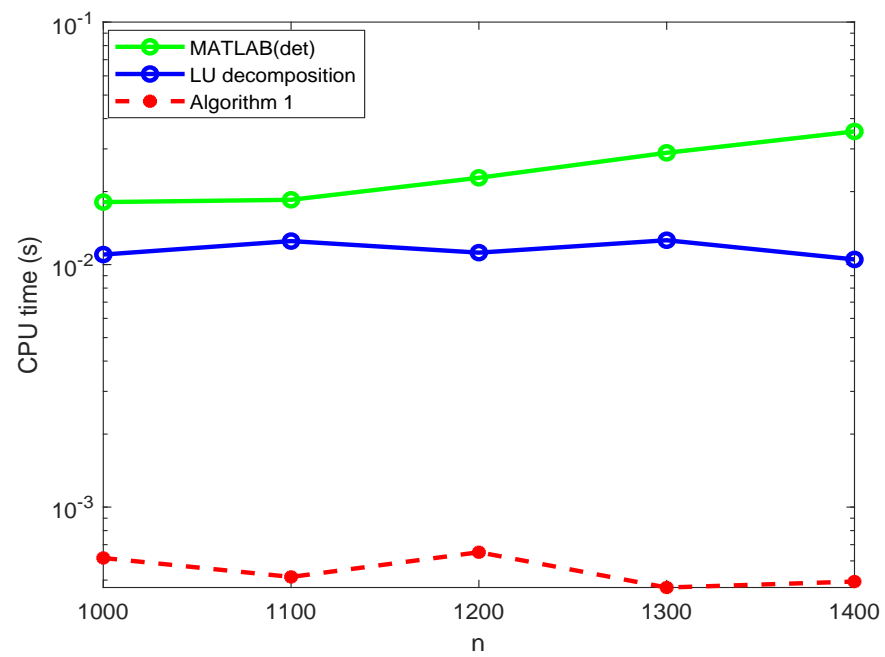

Figure 1: The CPU time comparison of calculating the determinant. 


\section{References}

[1] Y. P. Zheng, S. Shon, Exact determinants and inverses of generalized Lucas skew circulant type matrices, Appl. Math. Comput. 270, 105-113. (2015)

[2] X. Y. Jiang, K. Hong, Z. W. Fu, Skew cyclic displacements and decompositions of inverse matrix for an innovative structure matrix, J. Nonlinear Sci. Appl. 10, 4058-4070. (2017)

[3] J. T. Jia, Numerical algorithms for the determinant evaluation of general Hessenberg matrices, J. Math. Chem. 56(1), $247-256$. (2018)

[4] J. T. Jia, S. M. Li, New algorithms for numerically solving a class of bordered tridiagonal systems of linear equations, Comput. Math. Appl. 78, 144-151. (2019)

[5] G. Meurant, A review on the inverse of symmetric tridiagonal and block tridiagonal matrices, SIAM J. Matrix Anal. Appl. 13(3), 707-728. (1992)

[6] B. Choudhury, Diffusion of heat in multidimensional composite spherical body, IMA J. Appl. Math. 78(3), 474-493. (2013)

[7] P. L. Giscard, S. J. Thwaite, D. Jaksch, Evaluating matrix functions by resummations on graphs: The method of path-sums, SIAM J. Matrix Anal. Appl. 34(2), 445-469. (2013)

[8] A. J. Amar, Modeling of One-Dimensional Ablation with Porous Flow using Finite Control Volume Procedure (Master's Thesis), North Carolina State University, Raleigh, 2006.

[9] A. J. Amar, B. F. Blackwell, J. R. Edwards, One-dimensional ablation using a full newton's method and finite control volume procedure, J. Thermophys. Heat Tr. 22, 71-82. (2008)

[10] L. Losonczi, Eigenvalues and eigenvectors of some tridiagonal matrices, Acta Math. Hung. 60, 309-332. (1992)

[11] C. M. da Fonseca, The eigenvalues of some anti-tridiagonal Hankel matrices, Kuwait J. Sci. 45, 1-6. (2018)

[12] C. M. da Fonseca, V. Kowalenko, Eigenpairs of a family of tridiagonal matrices: three decades later, Acta Math. Hung. 160, 376-389. (2020)

[13] C. M. da Fonseca, J. Petronilho, Explicit inverse of a tridiagonal $k$-Toeplitz matrix, Numer. Math. 100, 457-482. (2005)

[14] C. M. da Fonseca, F. Yılmaz, Some comments on $k$-tridiagonal matrices: Determinant, spectra and inversion, Appl. Math. Comput. 270, 644-647. (2015)

[15] A. Yalçiner, The LU factorizations and determinants of the $k$-tridiagonal matrices, Asian-Eur. J. Math. 4(1), 187-197. (2011)

[16] J. T. Jia, T. Sogabe, M. El-Mikkawy, Inversion of $k$-tridiagonal matrices with Toeplitz structure, Comput. Math. Appl. 65, 116125. (2013)

[17] J. T. Jia, S. M. Li, On the inverse and determinant of general bordered tridiagonal matrices, Comput. Math. Appl. 69, 503-509. (2015)

[18] J. T. Jia S. M. Li, Symbolic algorithms for the inverses of general $k$-tridiagonal matrices, Comput. Math. Appl. 70, 3032-3042. (2015)

[19] R. A. Usmani, Inversion of a tridiagonal Jacobi matrix, Linear. Algebra Appl. 212(213), 413-414. (1994)

[20] M. Anđelić, C. M. da Fonseca, Some determinantal considerations for pentadiagonal matrices, Linear Multilinear A. 1-9. (2020) DOI: 10.1080/03081087.2019.1708845

[21] J. T. Jia, S. M. Li, On determinants of cyclic pentadiagonal matrices with Toeplitz structure, Comput. Math. Appl. 73, $304-309$. (2017)

[22] J. T. Jia, S. M. Li, An efficient numerical algorithm for the determinant of a cyclic pentadiagonal Toeplitz matrix, Comput. Math. Appl. 74, 2992-2999. (2017)

[23] J. T. Jia, On a structure-preserving matrix factorization for the determinants of cyclic pentadiagonal Toeplitz, J. Math. Chem. 57(8), 2007-2017. (2019)

[24] M. E. A. El-Mikkawy, A new computational algorithm for solving periodic tri-diagonal linear systems, Appl. Math. Comput. 161, 691-696. (2005)

[25] J. T. Jia, A breakdown-free algorithm for computing the determinants of periodic tridiagonal matrices, Numer. Algorithms 83, 149-163. (2020)

[26] M. A. El-Shehawey, Gh. A. El-Shreef, A. Sh. Al-Henawy, Analytical inversion of general periodic tridiagonal matrices, J. Math. Anal. Appl. 345, 123-134. (2008)

[27] T. Hopkins, E. Kılıç, An analytical approach: Explicit inverses of periodic tridiagonal matrices, J. Math. Anal. Appl. 335, 207226. (2018)

[28] J. T. Jia, Q. X. Kong, A symbolic algorithm for periodic tridiagonal systems of equations, J. Math. Chem. 52(8), $2222-2233$. (2014)

[29] M. Chawla, R. Khazal, Parallel elimination method for 'Periodic' tridiagonal systems, Int. J. Comput. Math. 79(4), $473-484$. (2002)

[30] M. El-Mikkawy, A new computational algorithm for solving periodic tri-diagonal linear systems, Appl. Math. Comput. 161(2), 691-696. (2005)

[31] T. Sogabe, New algorithms for solving periodic tridiagonal and periodic pentadiagonal linear systems, Appl. Math. Comput. 202 (2), 850-856. (2008)

[32] K. Thomas, Fibonacci and Lucas Numbers with Applications, John Wiley \& Sons, 2001. 
[33] F. Z. Zhang, The Schur Complement and Its Applications. Springer Science \& Business Media, New York, 2006.

[34] B. S. Zuo, Z. L. Jiang, D. Q. Fu, Determinants and inverses of Ppoeplitz and Ppankel matrices, Spec. Matrices 6, 201-215. (2018)

[35] G. H. Golub, C. F. Van Loan, Matrix Computations. Third ed.; The John Hopkins University Press, Baltimore, 1996.

[36] K. H. Rosen, Discrete Mathematics and Its Applications. Sixth ed.; New York: McGraw-Hill, 2011. 